University of Nebraska - Lincoln

DigitalCommons@University of Nebraska - Lincoln

To Improve the Academy

Professional and Organizational Development

Network in Higher Education

1997

\title{
The Pedagogical Colloquium: Taking Teaching Seriously in the Faculty Hiring Process
}

Pat Hutchings

Follow this and additional works at: https://digitalcommons.unl.edu/podimproveacad

Part of the Higher Education Administration Commons

Hutchings, Pat, "The Pedagogical Colloquium: Taking Teaching Seriously in the Faculty Hiring Process" (1997). To Improve the Academy. 379.

https://digitalcommons.unl.edu/podimproveacad/379

This Article is brought to you for free and open access by the Professional and Organizational Development Network in Higher Education at DigitalCommons@University of Nebraska - Lincoln. It has been accepted for inclusion in To Improve the Academy by an authorized administrator of DigitalCommons@University of Nebraska - Lincoln. 
Hutchings, P. (1997). The pedagogical colloquium: Taking teaching seriously in the faculty hiring process. In D. DeZure (Ed), To Improve the Academy, Vol. 16 (pp. 271-294). Stillwater, OK: New Forums Press and the Professional and Organizational Development Network in Higher Education. Key Words: Persomel Selection, Peer Evaluation, Faculty Role, Departments, Collegiality, Organizational Climate, Teaching Skills.

\section{The Pedagogical Colloquium: Taking Teaching Seriously in the Faculty Hiring Process}

\section{Pat Hutchings}

AAHE Teaching Initiative

American Association for Higher Education

In an effort to make teaching and learning more central, a growing number of campuses are adopting some form of the "pedagogical colloquium," a strategy proposed by Lee Shulman, President of the Carnegie Foundation for the Advancement of Teaching, in the context of a national project on the peer review of teaching. The purpose of the pedagogical colloquium is to create an occasion for examining and assessing the teaching skills and potential of faculty job candidates. Different models are now evolving, from formal presentations parallel in nature to the research colloquium commonly expected of job candidates, to more informal discussions of pedagogy, sometimes in combination with other strategies, such as teaching demonstrations. The pedagogical colloquium has the potential to make teaching more important in hiring decisions and to prompt important departmental campus conversation about expectations of faculty in the teaching arena, but it also raises a number of difficult issues. In this article, Pat Hutchings describes three emerging models, analyzes 
issues, and looks ahead to next steps in making the pedagogical colloquium a route to a more scholarly conception of teaching.

For the past several years, I have been involved in a national project entitled, "From Idea to Prototype: The Peer Review of Teaching." Housed at the American Association for Higher Education (AAHE), and undertaken in partnership with Lee Shulman at Stanford, the project entails work by a group of campuses (originally twelve and now sixteen) seeking to develop strategies through which faculty can make their teaching, like research, public and available to one anotherbe it for reflective discussion and improvement or for more formal evaluation linked to institutional rewards.

A premise of the project, from its beginning in January 1994, has been the need to break out of the box that equates the peer review of teaching exclusively with classroom observation. Accordingly, participating faculty and pilot departments have developed a wide range of strategies, suited to various occasions and purposes, including teaching circles, classroom visits, the development of course and teaching portfolios, and a number of others, all described, through reports by faculty using them, in a recent AAHE publication (Hutchings, 1996), Making Teaching Community Property: A Menu for Peer Collaboration and Review. One additional strategy that many faculty have been excited about - and the subject of this essay -is the pedagogical colloquium, an occasion aimed at getting better evidence about teaching effectiveness into the faculty hiring process and doing so in ways that foster a view of teaching as serious scholarly work.

To learn more about how the pedagogical colloquium can be most useful-both for making decisions about hiring and also for raising the level of attention to teaching - the American Association for Higher Education invited a group of twenty faculty to assemble this past February, in Palo Alto, California. Members of the group (see Appendix) were, with a few exceptions, already experimenting with the "ped colloq" (as we began calling it); some also brought to the meeting an interest in preparing their own graduate students for pedagogical colloquia and other occasions designed to assess teaching at the point of hire. 
The meeting spanned two days, during which we: (1) reported on our respective campus experiences with the pedagogical colloquium, as adapted to various contexts (and it was clear that adaptation was a key ingredient in success); (2) discussed more cross-cutting issues raised by the pedagogical colloquium; and (3) identified next steps for pursuing our interest in faculty hiring as a point of leverage for making teaching more central to academic culture. I have organized this essay around the same three areas, with gratitude to the meeting participants, whose experiences and insights constitute the bulk of what follows. But first a bit about the idea of the pedagogical colloquium, which brought us together.

\section{The Idea of the Pedagogical Colloquium}

The potential power of the pedagogical colloquium was first proposed several years ago by Lee Shulman, the Charles E. Ducommun Professor of Education at Stanford University. Speaking to a plenary session of AAHE's 1993 Conference on Faculty Roles and Rewards, Shulman (1993, p. 7) argued that if teaching is to be taken more seriously in higher education, if it is to be treated as scholarly, intellectual work and not just as a "load," institutions need to "change their advertising"-letting it be known that candidates for faculty positions will be required to offer "two colloquia. In one colloquium, they will describe their current research-the usual research colloquium. In the second, which we'll call the pedagogical colloquium, they will address the pedagogy of their discipline,...by expounding on the design of a course, showing systematically how this course is an act of scholarship in the discipline, and explaining how the course presents the central issues in the discipline and how in its pedagogy it affords students the opportunity to engage in the intellectual and moral work of the discipline." As one participant in the AAHE project on peer review later put it, the pedagogical colloquium is "the missing half of the job talk."

Paying attention to teaching in the faculty hiring process is not, of course, a new idea; and some campuses have a long tradition of assessing candidates' teaching competence or potential. But the fact is that many searches do not attend to teaching - not in any rigorous 
or thorough way. We have all heard stories about job interviews that touch on teaching only in a final, casual question: "Oh, by the way, what would you like to teach?" And we know, too, how ready many search committees are to assume that the candidate who can deliver a well crafted research colloquium can also teach effectively. AAHE's interest in the pedagogical colloquium is not, then, to deny that models for assessing teaching in the hiring process are already "out there," but rather to uncover and promote more such models.

As one of those models, the pedagogical colloquium would offer several advantages. First, and most obviously, it would, over time, begin to shift the composition of the department by promoting the hiring of faculty more effective at and more interested in teaching; indeed, several of the departments reporting at the Palo Alto meeting (and in previous literature), confirm this effect, noting that the pedagogical colloquium can indeed change decisions about whom to hire. Richard Roberts, a faculty member in the Stanford history department, points out (1996, p. 84) that his department's adaptation of the pedagogical colloquium (described below) "provided important information that the department considered in making appointments. For instance, there was one candidate who gave a good -a very good-job talk; the research was really very well honed. But when it came to talking about teaching, it became clear that this candidate had put very little time into thinking about teaching. The search committee took this information into consideration when advancing a finalist to the department."

Second, the pedagogical colloquium would bring faculty colleagues into substantive conversation about teaching and learning. That is, having attended candidates' pedagogical colloquia, faculty must come together to discuss and debate what they have heard-and in the process grapple with departmental expectations for teaching in a way they may not otherwise have occasion to do. Heidi Bymes (1995, p. 10), a member of the German department at Georgetown University reported on this effect in an $A A H E$ Bulletin piece: "...in a curious way," she writes, "what we had started out focusing on-namely the research colloquium-engendered less discussion; the center of our deliberations was actually how the candidate approaches the field, as revealed through issues of teaching and learning...[T] he new collo- 
quium model reinforced a sense of community" based on teaching and learning.

Finally, the pedagogical colloquium would, over the longer term, help shift the emphasis of graduate programs by signaling an insistence on the "demand side" that newly minted Ph.D.s (and maybe not so new ones as well) be prepared for their teaching roles as well as for research. It's important to acknowledge, here, that the "demand side" is a pretty weak one in many fields these days; the sad state of the academic job market is all too well known. On the other hand, the hiring of faculty, infrequent as it may be in some settings, is an undeniably high-stakes activity-consuming considerable time and money, and affecting not only individual careers but also the character of the department itself - often, for years to come. Moreover, there is today a growing national interest in better preparation of graduate students for their roles as teachers (for instance through the Pewfunded project, "Preparing Future Faculty," and in the biennial conference on the education and employment of graduate students), making this a right time to help hiring campuses be more thoughtful about how to assess that preparation (and encourage more of it).

\section{Models of the Pedagogical Colloquium}

Where things get interesting is in the detail of how to design and conduct a pedagogical colloquium that will maximize the potential benefits of the strategy. For starters, one must decide, for instance, what is the right "assignment" for candidates. What, exactly, should hiring departments ask candidates to speak about? Lee Shulman proposed three possible answers to this question in an essay several years ago $(1995$, p. $7-8)$. The pedagogical colloquium might focus, he said, on: (1) a course narrative, exploring why the course is shaped and structured as it is; (2) essential ideas or concepts in the field that are "devilishly difficult to teach... or rather they're easy...to teach but hard for students to learn"; and (3) pedagogical dilemmas endemic to the teaching of the field, such as finding the right balance between depth and breadth in a history survey course. But how have these models worked out in actual campus experience? What other "assignments" have proved to be useful? And what artifacts or evidence (a 
syllabus? videotape? course portfolio?) should candidates be invited to share with the department-if any?

There are also questions about the character of the occasion: Who should attend the pedagogical colloquium? Faculty certainly. What about graduate students? Undergraduate? In what setting is the colloquium best conducted? A formal presentation, closely modeled on the research colloquium? A more informal, interactive occasion? How long should the occasion last and where is it best placed in the longer timeframe of the campus visit?

Needless to say, there are no "right" answers to the above questions, and the Palo Alto group spent much of its time uncovering variations and options. Indeed, meeting participants continually emphasized the need to adapt the basic idea of the pedagogical colloquium to the discipline or field of the study, the department or program context, and the character of the institution. Nevertheless, many of the examples fell into three general categories which can, I think, be usefully illustrated with three particular examples.

\section{The Pedagogical Colloquium as Scholarly Presentation: Educational Studies Program, University of Michigan}

Several years ago, faculty in the Educational Studies Program at the University of Michigan made a decision to make teaching more central to departmental policy and practice. As one aspect of this shift, they began looking at the process of faculty hiring, seeing it as an occasion to signal to candidates (and reinforce for themselves) the centrality of teaching to the field. The chosen vehicle for doing so, as explained by department chair Ronald Marx at the AAHE meeting, was a pedagogical colloquium shaped very much on the model of the formal research presentation. Faculty members on the first search committee to use the pedagogical colloquium discussed different approaches and decided to treat the occasion as a formal part of the hiring process in order to signal the central importance of teaching to both the candidate and the rest of the department. An informal pedagogical colloquium coupled with a formal research colloquium, it was argued, would suggest that the former did not require the attention to 
planning and argumentation that the latter does. As such, different approaches to the two presentations, rather than signaling the importance of teaching, would do just the opposite. That is, it would show that teaching "really isn't as important as research."

This decision to establish a formal pedagogical colloquium necessitated a number of changes in the search process. First, the campus visit was extended to two full two days to allow time for the pedagogical colloquium. Secondly, candidates were asked to send to the search committee not only copies of their scholarly research but also instructional materials they had developed: syllabi, assignments, assessments - materials that might constitute a teaching portfolio (though the department does not require a portfolio per se). And, just as originally envisioned by Lee Shulman, candidates were asked to prepare and deliver two colloquia, the traditional research talk and the pedagogical colloquium.

The pedagogical colloquium is given a full hour and a half. During the first half (or so) of this time, the candidate is to present a sustained and carefully developed scholarly argument about the teaching of the field; the second half is to be an interactive engagement with department members, managed in whatever way the candidate sees fit. Typically, this second section entails a lively Q\&A session in which faculty have a chance to probe the candidate's deeper thinking about his or her teaching, an occasion, as Marx reported to the group, that has turned out to be especially useful and revealing when discussion is concrete and practical rather than grandly philosophical. If possible, the pedagogical colloquium is scheduled before the candidate's lunch with graduate students, with the notion that it's fodder for a lively discussion. In fact, graduate students have reported that their lunches with candidates are now far more productive. When the lunch follows the pedagogical colloquium, the students are able to ask more penetrating questions, helping them to get a much better sense of candidates' approaches to undergraduate and graduate education, and a better view of how candidates use their knowledge of the field to reason about matters of teaching.

Michigan's program has now employed the pedagogical colloquium for two years, in twelve searches, and Marx shared several lessons from that experience with the Palo Alto group. For one, the 
new occasions have created additional demands on people's energy and time; in particular, it poses a challenge in terms of "getting an audience out" - a challenge the department has addressed by identifying a "point person" to take responsibility for putting an appropriate audience together. A second lesson is that while there's some consensus about criteria through which a candidate's research program can be evaluated, criteria for judging the pedagogical colloquium havenot surprisingly - been more difficult to identify, though Marx believes they are now, slowly, starting to evolve.

In sum, Marx concluded, the department is beginning to find that candidates' scholarly abilities are illuminated by the pedagogical colloquium just as much as by the research colloquium. Especially as faculty and graduate students learn to use the pedagogical colloquium as a legitimate component of the hiring process, they are increasingly pointing to the pedagogical colloquium as a source of information and insight equal in value to the research colloquium.

\section{The Informal Discussion of Teaching and Curriculum: Department of History, Stanford University}

The Stanford history department started experimenting with the pedagogical colloquium after professor Richard Roberts attended the week-long opening institute of the AAHE Peer Review of Teaching project in June of 1994, seeing an opportunity to make teaching more central to his department's culture by paying greater attention to it in the faculty hiring process. Roberts' colleagues were, he told us, skeptical at first, but they agreed to try an adaptation of the pedagogical colloquium on an experimental basis - and with the understanding that it would take the form of an informal discussion rather than (as at Michigan) a more formal scholarly presentation. This format seemed to faculty a better match with what could reasonably be expected of new Ph.D.s in the field, Roberts noted, and one more likely to reveal the candidate's capacity for collegiality around teaching and learning.

Now, some three years later, the "informal discussion about teaching and curriculum" is fully built into the department's hiring process. Each candidate is asked to give a formal research presenta- 
tion, but the department, in the person of the chair of the search committee, also instructs candidates to come prepared to talk about teaching -and, if they wish, to bring along materials related to their teaching - for example a syllabus or course description. The search committee chair often sends the candidate the short description of the department's "informal discussion" published in the AAHE Bulletin (Roberts, 1996) in order to familiarize the candidate with the department's practice.

The discussion is usually scheduled for the morning following the formal scholarly presentation. Faculty members, members of the search committee, and graduate students attend. Sometimes the session is scheduled over lunch, to promote the kind of informality and collegial exchange the department is interested in seeing. Typically, the chair of the search asks everyone around the table to introduce themselves, so the candidate knows the mix of the group, then asks the candidate an opening question about, say, the kind of courses she or he anticipates teaching, and how those courses might be organized and focused. There may be questions, as well, about how to adapt a course taught on the semester system to the quarter system employed at Stanford; there may be questions about how the candidate would teach a research-methods course, or a lecture course. As at Michigan, department members have found that the more telling discussion is the more concrete, grounded one. For instance, hearing how the candidate would approach a key text or historical problem is more useful than hearing that he or she is in favor of active learning.

Though Roberts is concerned about the need to follow up more systematically on the "informal discussion" in other aspects of departmental policy and practice, he sees progress as well. For one thing, attendance at the event has significantly increased, from six or seven in the first instance to twenty in a recent search for a senior appointment.

Secondly, it has become clear that different occasions provide different windows on candidates' strengths and weaknesses. The formal, research-based job talk, which the candidate is very much prepared for, gives the department a look at the candidate's ability to perform in a situation he or she largely controls. In contrast, the informal discussion, in Roberts' judgment, provides an opportunity to 
assess the candidate's quality of mind because topics range more broadly and less predictably. Indeed, candidates who don't do as well in their formal job talk have on occasion "redeemed themselves" in the informal discussion about teaching and curriculum, changing the department's decision about whom to hire.

The informal discussion has also been important, according to Roberts, in sending a signal to candidates that department membersand even the most senior faculty - take teaching seriously. In this sense, the department reaped double benefits from its recent informal discussion about teaching with a senior historian being considered for a position in the department. First, it provided the department members with a sense of how committed the candidate was to teaching both graduate and undergraduate students; second, it sent a message to the candidate that the faculty takes its teaching very seriously. In an informal conversation with the candidate later in the day, department members learned that the candidate was clearly pleased by this demonstration of interest in teaching.

The same signal is clearly received by graduate students in the department, who are, having attended these events, encouraged to see teaching as more central to their role - now (they are required to teach four times in the history department) and in the future. In short, the department's adaptation of the pedagogical colloquium is a step toward changing expectations about academic life and values.

\section{A Mixed Demonstration/Presentation Model of the Pedagogical Colloquium: Department of English, Kent State University, Stark Campus}

Talking about teaching is not a new thing on the Stark Campus of Kent State University, Virginia Carroll reported to the Palo Alto group, and the department of English-like other units of the campus - has long required a teaching demonstration of job candidates. Recently, however, the department agreed to add to the mix a pedagogical colloquium designed to give candidates an opportunity not only to "do teaching" but also to clarify the thinking behind their practice. 
In advance of the campus visit, candidates are apprised of the importance of teaching to the department's mission. The search committee sends candidates the undergraduate catalog, memos about changes in the major, the campus viewbook, and other documents focusing on the educational program. Candidates are also alerted to the fact that they should arrive prepared to make a 20 minute presentation, addressing four questions, as follows:

- What assumptions do you make about students, and how do these assumptions inform your teaching?

- What are the reasons for success in the best class you have ever taught?

- What guided your choices in planning and carrying out today's teaching demonstration?

- What are the connections between your teaching and your research?

Once on campus, the candidates' day may begin with a campus tour and assorted "orientation" activities. They then give an actual teaching demonstration to an identified class, on a topic chosen by the candidate in consultation with the search committee chair. Candidates in the most recent search, for example, were asked to teach a section of the second-semester composition course, which uses imaginative literature as the basis for student writing; they had the opportunity to assign a work of literature of their choice for the session they taught, and to move from formal presentation to discussion and actual writing about the assignment. The pedagogical colloquium, organized around the four questions sent in advance, follows, allowing for questions about the teaching demonstration, as well as broader concerns. (Faculty from all disciplines are invited to attend the teaching demonstration, though some attend the pedagogical colloquium but not the demonstration).

As in the Stanford history department, participation in the colloquium is on the rise at Kent, with more faculty attending each time. Moreover, Carroll reports that the quality of discussion with candidates has improved over time, with faculty asking in this public forum the kind of sophisticated, in-depth questions that might previously have been asked only in closed session with the search committee. Interestingly, judgments of the teaching demonstration have usually 
been reinforced rather than contradicted by the pedagogical colloquium, but either of the two strategies would, Carroll argues, be less helpful alone than in tandem with the other.

Kent's "mixed model" will be assessed at the end of this (1996-97) academic year, using information gleaned from faculty who attend the colloquia as well as suggestions and reflection from the candidates themselves. The department is especially interested in studying the effectiveness of the method as a predictor of later success in the classroom.

\section{Issues Raised By The Pedagogical Colloquium}

\section{Setting Reasonable Expectations of New Faculty}

An issue very much on the minds of all of us at the Palo Alto meeting was the added demand on job candidates-especially on graduate students newly entering the job market—posed by the pedagogical colloquium As one person put it, it is unfair to expect graduate students to perform in occasions like those described above "without appropriate preparation and practice in their home departmentswhich is clearly the exception rather than the rule at present." Moreover, there was concern that newly minted Ph.D.s are expected to live up both to the traditional (and perhaps even escalating) high standards for research and the newer standards related to teaching. The point here, as I understood it, was not to argue against using the pedagogical colloquium but to urge mindfulness about setting reasonable expectations-which may vary, for instance, by field. In English and composition, and in foreign languages, many graduate students have occasions to teach, and pedagogy is a subject of explicit scholarly inquiry, making some version of the pedagogical colloquium a meaningful and relevant expectation. In chemistry, however (as chemist David Malik told us at the Palo Alto meeting), graduate training focuses almost exclusively on research; similarly, in journalism and mass communications programs (as evidenced by a survey of Ph.D. programs in the field conducted by a Palo Alto participant Jeremy Cohen), "doctoral students generally are neither required to teach their own classes, nor to serve as teaching assistants" $(1997$, p. 34). In such 
circumstances, the pedagogical colloquium becomes more problematic.

The flip side of this concern about expectations is, of course, a hope that as occasions like the pedagogical colloquium become more common and expected parts of the job search process, graduate programs will take steps to prepare students more fully. Indeed, a number of folks around the table in Palo Alto were attempting to put in place programs to do just that: help graduate students become more skilled, thoughtful, and articulate about teaching and learning. Mark Gonnerman, a graduate student in religious studies at Stanford, reported on a four-credit graduate-level course focused on the pedagogy of the field, which he helped organize in his department. Aimed at bringing faculty and graduate students into conversation about teaching, and raising awareness of "the scholarship of teaching," the course consists of a series of seminars in which faculty from the program $\rightarrow$ different one each week-talk about a course of their choosing, focusing on how the course has changed over time and what he or she has learned about teaching in the process of that evolution (Shulman's course narrative model, if you will). The course, says Gonnerman, is manageable in terms of faculty commitment and graduate student schedules, and it has "shown people that these issues are intellectually compelling." Moreover, the success of the course recently led the department to adopt the pedagogical colloquium for use in its hiring process. Gonnerman's report was one of the prompts that led Susan Rava, a faculty member in foreign languages at Washington University, to follow up on the Palo Alto meeting by proposing a final stage of preparation for graduate students in her setting, focused on "teaching requirements of hiring institutions, preparation of a teaching philosophy statement; preparation for teaching and teaching conversations during on-campus interviews..."

Programs like Rava's and Gonnerman's are, it is important to say, currently the exception rather than the rule, and there was strong sentiment around the table in Palo Alto about the need to calibrate expectations related to teaching in light of current realities. The pedagogical colloquium asks candidates to engage in a kind of discourse for which there's little tradition. At the very least, we owe candidates clear statements about the purpose of the event, how the 
occasion will unfold, and what if any specific expectations the department holds.

\section{Ensuring Fair and Appropriate Judgments}

An issue that arose early on during the Palo Alto meeting was about the difficulty of judging candidates' pedagogical colloquia presentations and interactions. For starters, this is an issue about standards because few departments or campuses have a clear consensus about what constitutes effective teaching; thus, we may be making very high-stakes decisions without a clear, shared sense of the appropriate criteria for judgment (though it should be said that this problem exists quite independent of the introduction of the pedagogical colloquium).

Secondly, the issue is one of expertise, and whether, in fact-even where standards may be clear-faculty are able to make judgments they can be confident about. To put this point in the negative, a worry about the table was that faculty who themselves have little training in teaching, and not much vocabulary for talking about it, are then asked to judge the practice and ideas of another teacher-whose style and approach might be antithetical to their own. Is there not, we asked ourselves, a danger that judgments will be based on personal biases more than on any solid knowledge base? Might a group of faculty, well entrenched in (and skilled at) lecture, find a candidate interested in more "active" learning techniques, more alternative strategies, unacceptable? (Indeed, something very like this happened in one of the reporting departments.) In short, we found ourselves worrying that the pedagogical colloquium might require more pedagogical training and expertise among faculty than can currently be found in most settings.

Like the previous issue, this one too has a flip side, which lies in a hope that through repeated exercises of judgment, faculty and departments will develop greater sophistication about their conception of effective teaching and how to recognize it. And, as noted in the account of Kent State's "mixed model" above, we did in fact hear stories of an increasingly sophisticated process of inquiry and judgment. Perhaps it's useful to say, as well, that judgments about teaching 
are never going to be the kind of "high science" in which all margin of error is eliminated. What will move us ahead in the short run is neither a checklist of criteria (almost certain to be reductive) nor an expectation that faculty will have extensive grounding in the literature of pedagogical theory and practice (nice but not likely in most settings). What's needed, rather, and what the pedagogical colloquium can help provide, is the chance for faculty to be part of a community of discourse in which they can, over time, develop increasingly sophisticated capacities for expert judgment.

\section{Appropriately Valuing Various Sources of Evidence}

The premise of the Palo Alto meeting was not that the pedagogical colloquium is the only or even the best way to assess teaching in the hiring process; its premise was that the "ped colloq" might tell us things that other strategies may not and that it might, therefore, usefully complement and strengthen others aspects of the search process. That said, a good deal of discussion focused on the relative merits of different sources of evidence about teaching. Many campuses and departments have, in the spirit of "authentic assessment," traditionally required candidates to give a teaching demonstration, and many are adamant about the importance of this kind of first-hand look at classroom behavior, style, rapport with students, and the like (and skeptical about whether a candidate's capacity to talk well about teaching is related to actual practice over time). Meanwhile, advocates of the pedagogical colloquium argue for its merits in revealing the candidate's "pedagogical thinking" rather than relying on a one-shot classroom observation (a method famous for its unreliability in summative peer review contexts).

The point here is not that one method is intrinsically better than the other. The point, rather, is that we need to be aware of what we can and cannot learn from various strategies; that no single piece or source of evidence can paint the whole picture... Indeed, a question for further exploration is about the larger menu of strategies for assessing teaching in the hiring process: What, for instance, does one learn (and not) from having candidates teach an actual class? A simulated class? Meet with students? What distinctive advantages and 
limits does the pedagogical colloquium have among this larger menu of approaches? And what is gained if we add to the mix a portfolio that candidates might submit? How are our efforts to assess teaching related to our examination of the candidate as a scholar and researcher? And, finally, what is the right combination of evidence for making judgments we feel confident about and that stand up over time in light of subsequent performance?

\section{Alignment with Departmental Policy and Practice}

Though our Palo Alto meeting was focused originally and explicitly on questions of faculty hiring, the group talked long and hard about other aspects of department or campus culture. We were concerned, that is, about scenarios in which faculty would be hired on the basis (in part) of teaching competence and then find department culture (for instance, and perhaps most notably, policies related to promotion and tenure) squarely focused on research. The issue, as one person at the table put it, is "institutional integrity." If the pedagogical colloquium is a practice we want to promote, we must do so with attention to what follows it; otherwise, as another participant put it, "we put new faculty in the crossfire between old and transitional ideas" about faculty roles and rewards.

This said, one comes to the next question, about what, exactly, the campus or department might do, in terms of departmental practice and policy beyond the point of hire, to deliver on the tacit promise of the pedagogical colloquium. One thinks for instance of new faculty orientation, of mentoring, of the review of probationary faculty, of the tenure and promotion process, or post-tenure review.

This essay is not the place to address this full list of topics, but it is useful, I think, to note that one step in the right direction, which generated real enthusiasm among Palo Alto meeting participants, is to think about adapting the pedagogical colloquium itself to departmental purposes and occasions beyond the point of hire. Indeed, a case in point was provided by Mary Gendernalik Cooper, who reported on the pedagogical colloquium as a faculty development strategy at Mary Baldwin College, where Cooper directed a Master of Arts in Teaching program before moving to her present position at Augusta State. 
A graduate level teacher licensure program grounded in the arts and sciences, the Mary Baldwin MAT program poses a special challenge in that the program has virtually no faculty of its own; no one's primary instructional responsibilities (except the director's) are in the MAT program. With this in mind, Cooper set about to find a professional development approach that would help faculty understand the program's emphasis on inquiry-based teaching and learning. The approach she chose was a series of pedagogical colloquia, conducted through a series of discussion dinners for MAT faculty throughout the academic year. The series was built around the three models proposed by Lee Shulman (and briefly noted above): the course narrative model, the essential ideas and concepts model, and the dilemma-based model. Each of the three models was presented by a faculty volunteer, who then engaged other participants in thinking through similar questions and issues in their own teaching.

Cooper reports (1997) that participants found all three models useful for stimulating substantive conversations about teaching and learning. The experience also reduced isolation and increased understandings of larger program goals. Interestingly, there was enthusiasm as well for additional faculty development activities organized around the three models. When asked whether the model could be adapted for faculty performance reviews, participants were less certain but interested in further exploration of the ideas.

\section{Next Steps on Campus and Beyond}

The final afternoon of the Palo Alto meeting focused on what it would take to advance the concept and practice of the pedagogical colloquium. Here, briefly, are a few useful next steps for individual campuses and (in the final instance) at a national level:

1. Start a conversation among relevant parties on your campus about how teaching is currently assessed during the hiring process. This worked well (and rather easily) at the University of Wyoming, where the dean of arts and sciences called a meeting of all chairs whose departments would be hiring in the subsequent year, inviting folks simply to talk about what they do (and providing everyone with a copy of an article about the pedagogical colloquium to 
spur further thinking). No surprise: practices were found to vary widely from department to department: What makes sense in the English department differs from what makes sense in, say, the chemistry department. Nevertheless, participants in the discussion (about 15 attended) reported that the discussion gave them new ideas and caused useful reflection about current practices and possible improvements.

2. Identify and circulate useful materials. As noted below, there's little literature on the faculty hiring process, but two articles on the pedagogical colloquium have now appeared in the $A A H E$ Bulletin, which is not copyrighted and can be duplicated and passed around on campus (Bymes, 1995: Shulman, 1995). A bit of reconnaissance would no doubt turn up other useful items as well.

3. Plan to evaluate the process. Departments attempting to augment their hiring process with further attention to teaching would do well (for themselves and others) to monitor their efforts. A useful role for faculty development staff might, in fact, be to conduct focus groups or interviews with those involved (members of the search committee, attendees at the pedagogical colloquium, and candidates themselves) about what was useful and not. Case studies would be particularly worthwhile, and the campus might want to collect and disseminate a set of such to promote further refinements. Or take a cue from Lee Seidel at the University of New Hampshire, who shared with the Palo Alto group the results of a survey of current practice he conducted on his own campus.

4. Finally, on the national level, there's a need for much better information about what actually transpires in the faculty hiring process. A recently conducted literature search to see what is currently known (Seidel, 1997) reveals the paucity of information about the topic. Scholarly societies track employment trends, and some are exploring the hiring phenomenon in more fine-grained ways (see, for instance, papers by Cohen, Mangum, and Perlman, listed below), but there's clearly a need for more carefully collected baseline information about attention to teaching at the point of hire. Learning, for instance, that an increasing number of searches entail assessment of teaching competence would be a 
powerful nudge for graduate programs concerned about placing students. Additionally, such information might lead increasing numbers of departments and campuses to examine their own practices and seek ways (the pedagogical colloquium is only one) to take teaching more seriously in the hiring process and beyond.

\section{A Final Note}

I would welcome hearing from readers of this article who are using or interested in the pedagogical colloquium and other strategies for making teaching a more central component of the faculty hiring (and therefore graduate-student preparation) process.

\section{References}

Bymes, H. (1995, May). Faculty hiring: One department's experience. American Association for Higher Education Bulletin, 47, 9-10.

Cohen, J. (1997, Winter). Learning the scholarship of teaching in doctorate-granting institutions. Journalism \& Mass Communication Educator, 51, 27-38.

Cooper, M. G. (1997, July). The pedagogical colloquium as faculty development. Selected papers and awards from the 8th National Conference on College Teaching and Learning, Jacksonville, FL.

Hutchings, P. (1996). Making teaching community property: A menu for peer collaboration and peer review. Washington, DC: American Association for Higher Education.

Mangum, T. (1996, Fall). Identity and economics; or, the job placement procedural. Association of Departments of English Bulletin, No. 114, 19-24. This issue also contains other pieces about hiring in departments of English.

Perlman, B., et al. (1996, April). Applicants for a faculty position do not emphasize teaching. Teaching of Psychology, 23, 103-4.

Roberts, R. (1996, November). Adding teaching to the "job talk." American Association for Higher Education Bulletion 49, 3-6.

Roberts, R. (1990). The pedagogical colloquium: Focusing on teaching in the hiring process in the Stanford university history department. In P. Hutchings (Ed.), Making teaching community property: A menu for peer collaboration and peer review (pp. 83-85). Washington, DC: American Association for Higher Education.

Seidel, L. F. (1997, February). The faculty hiring process: Bibliography. Document shared with AAHE meeting on the pedagogical colloquium, Palo Alto, CA. Abstracts prepared by Robin Lyday and Mark Vosvick, Stanford University.

Shulman, L. S. (1993, November-December). Teaching as community property: Putting an end to pedagogical solitude. Change, 25, 6-7. 
Shulman, L. S. (1995). Faculty Hiring: The pedagogical colloquium: Three models. American Association for Higher Education Bulletin 47, 6-9.

Contact:

Pat Hutchings

Director, AAHE Teaching Initiative

Center for Teaching Excellence

O. Box 3334

University of Wyoming

Laramie, WY 82071

Phone: 307-766-4825; fax: 307-766-4822

E-mail: path@uwyo.edu

Pat Hutchings. As director of the Teaching Initiative at the American Association for Higher Education, Pat Hutchings works with faculty, administrators, and others to develop and foster a campus culture in which teaching and learning are talked about, inquired into, evaluated and rewarded. Prior to working at AAHE, Pat was a faculty member and chair of the English Department at Alverno College in Milwaukee, Wisconsin. She is currently a visiting professor at the University of Wyoming. 


\title{
Appendix \\ List of Palo Alto Meeting Participants \\ February 21-22, 1997 \\ Palo Alto-Stanford Holiday Inn
}

\author{
Jerry Berberet \\ Executive Director \\ Associated New American Colleges \\ Mark Booth \\ Associate Dean of Arts and Sciences \\ University of Wyoming \\ Virginia Carroll \\ Department of English \\ Kent State University-Stark Campus
}

Jeremy Cohen

Associate Dean, College of Communications

The Pennsylvania State University

Mary Gendernalik Cooper

Chair, Teacher Development Department

Augusta State University

\section{Ann Heiss}

Department of History

Kent State University-Main Campus

Mark Gonnerman

Graduate Student, Religious Studies,

Stanford University

\section{Tom Hatch}

Research Associate, Harvard Project Zero

Visiting Scholar, Stanford University 


\section{Pat Hutchings}

Director, AAHE Teaching Initiative

American Association for Higher Education

Deborah Langsam

Department of Biology

University of North Carolina-Charlotte

\section{John Layman}

Department of Physics

University of Maryland

\section{Robin Lyday}

Graduate Student, Education,

Stanford University

\section{David Malik}

Chair, Department of Chemistry ,

Indiana University-Purdue University Indianapolis

Michele Marincovich

Director, Center for Teaching and Learning

Stanford University

\section{Ronald Marx}

Chair, Educational Studies Program

University of Michigan

\section{Susan Rava}

Department of Romance Languages and Literature Washington University

\section{Eugene Rice}

Director, Fonum on Faculty Roles and Rewards

American Association for Higher Education

\section{Richard Roberts}

Department of History

Stanford University

\section{Lee Seidel}

Director, Teaching Excellence Program

University of New Hampshire 


\author{
Lee Shulman \\ Charles E. Ducommun Professor of Education \\ Stanford University \\ and President-designate, Camegie Foundation for the Advancement of Teaching \\ Mark Vosvick \\ Graduate Student, Education \\ Stanford University \\ Ellen Wert \\ Program Officer \\ The Pew Charitable Trusts \\ Thomas Wortham \\ Department of English \\ UCLA
}

